Case Report

\title{
Leiomyosarcoma : Case Report of a Rare Primary Smooth M uscle Tumour of the Thyroid Gland
}

\author{
Bhavika A. Kothari ${ }^{1}$, Aditya Godkhindi ${ }^{2}$, Rohan Shetty ${ }^{3}$, Vishwanath S. ${ }^{4}$ \\ ${ }^{1}$ Post graduate, ${ }^{2}$ Senior resident, ${ }^{3}$ Associate Professor, ${ }^{4}$ Assistant Professor, Department of General Surgery, Justice K.S. \\ Hegde M edical Academy, M angalore..
}

*Corresponding Author : Bhavika A. Kothari, post graduate, department of general surgery, Justice K.S. Hegde Hospital, M angalore.

Received

: 10.01.2017

Review Completed : 02.02.2017

Accepted

:03.02.2017

Keywords:

\begin{tabular}{|c|}
\hline Access this article online \\
\hline Quick Response Code \\
\hline
\end{tabular}

\begin{abstract}
Primary smooth muscle tumours of the thyroid gland are extremely rare neoplasms. There are concerns regarding the management of such disease due to its rarity. Around 22 cases have been reported in the literature till date. In this study, the case of a 63year old female patient who presented with a rapidly enlarging anterior neck mass over the period of 3 months is reported. The patient underwent thyroidectomy with tracheostomy and radiotherapy for the treatment of primary leiomyosarcoma of thyroid gland. Due to similar clinical presentation as anaplastic carcinoma, diagnosis of primary thyroid leiomyosarcomas is difficult and maybe misdiagnosed. Hence the combined assessment of clinical, imaging and pathological data is required.Immunohistochemical analysis is a gold standard for diagnosis. Diagnosis and treatment of the current patient with primary thyroid leiomyosarcomaand a comprehensive review of the relevant literature are presented herein
\end{abstract}

\section{Introduction}

Leiomyosarcomas are commonly seen in the pelvis, thegastrointestinal tract and the retroperitoneum. Sarcomas are thought to originate from the wall of blood vessels [1]. The etiology of primary thyroid leiomyosarcomas remains unclear, however, certain authors have postulated that it may originate from the smooth muscle in the vascular walls $(7,10,11-13)$. They account for $6 \%$ of the head and neck tumours, of which only $\mathbf{0 . 0 1 4 \%}$ of Leiomyosarcomas cases develop in the thyroid gland $(7,8,9,10,13,14)$, with an estimated 1year survival rate of $5-10 \%(10,12)$. Primary thyroid leiomyosarcomais an extremely rare soft tissue cancer. Studies relating to the management of these clinical cases are lacking. Although an improved multimodal approach is often necessary.The complexity of the procedure depends on tumour size and adjacent tissue/organ involvement. Adjuvant radiochemotherapy appears to be ineffective and a high mortality rate is observed. Thyroid leiomyosarcoma remains a fatal tumour, and innovative and more effective therapeutic strategies to improve management and outcomes are required. Primary thyroid leiomyosarcoma may be mistaken for other tumours, such as anaplastic ormedullary carcinomas. Diagnosis should be clarified by immunohistochemical staining methods when there is a tumour resembling spindle shaped malignant cells [4]. Together with anaplastic carcinoma they are the most aggressive variant of the thyroid cancers with a very poor prognosis [6] Primary thyroid leiomyosarcoma are more often reported in elder individuals and so far only 22 welldocumented cases have been reported in the world literature.Therefore, the diagnosis is difficult and requires numerous clinical, radiological, and pathological investigations

\section{Case Report}

A 65 year old female patient, presented to our hospital with history of sudden enlargement of swelling in front of the neck. Patient came with history of swelling in front of the 
neck since thirty years with a sudden increase in the size in the past three months, history of associated complaints of altered voice since one week, dysphagia to liquids then solids, tremors of right leg since 3 months. Also gives history of significant weight loss since 2 months. Clinical examination revealed the presence of large $14 \times 8 \mathrm{cms}$ swelling in front of the neck which moves up on deglutition. Endoscopic examination revealed extra luminal compression of the upper third oesophagus with pan gastritis. Videolaryngoscopy revealed left vocal fold palsy in adducted position. Thyroid hormone assay was within normal range. Ultrasound-guided fine needle aspiration cytology showed features suggestive of papillary carcinoma of thyroid. Computed tomographic scan revealed larger heterogenous mass involving bilateral lobes and isthmus with micro and macro calcifications within. Also, loss of fat plane with larynx present and mass displacing trachea and oesophagus with no retrosternal extension. The patient underwent total thyroidectomy and level 6 nodal clearance i.e superiorly upto hyoid bone, bilateral carotid sheath on either sides or inferiorly azygous vein.All nodes were cleared including fat. Also tracheostomy was done due to left vocal cord palsy and intraoperative findings involving infiltration into trachea and left recurrent laryngeal nerve. Histopathological features of specimen revealed undifferentiated carcinoma of thyroid gland involving tracheal mucosa and one cervical lymph node/satellite nodule. Hence further evaluation in the form of immunohistochemistry of specimen was done which was positive for smooth muscle antigen and gave the diagnosis of Primary thyroid leiomyosarcoma. Patient came for follow up after a month and received radiotherapy of 60 Gys in 40 fractions over 40 days

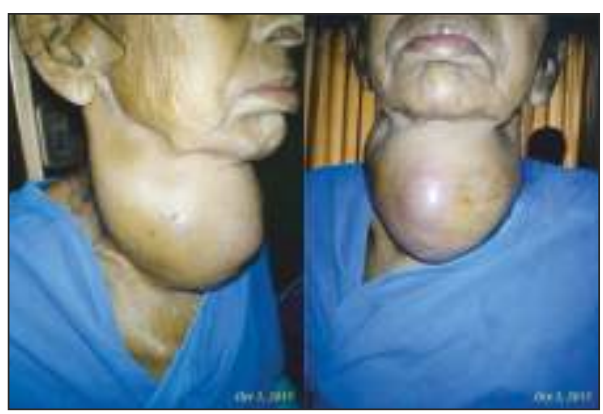

Preoperative Clinical Picture

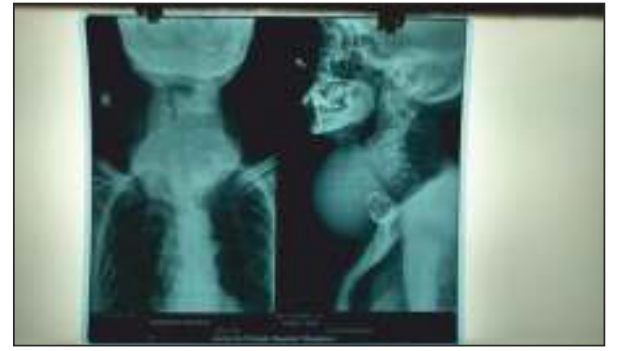

Preoperative X-Ray

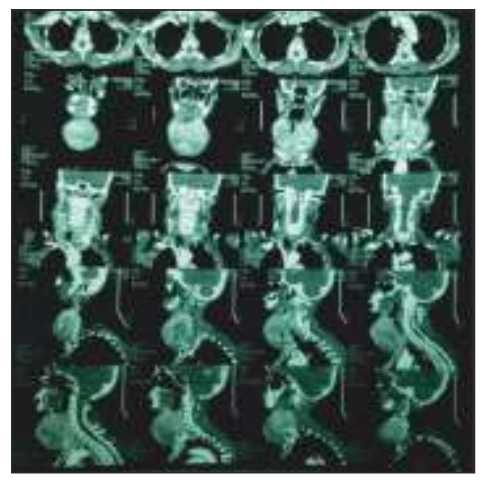

Preoperative CT Scan

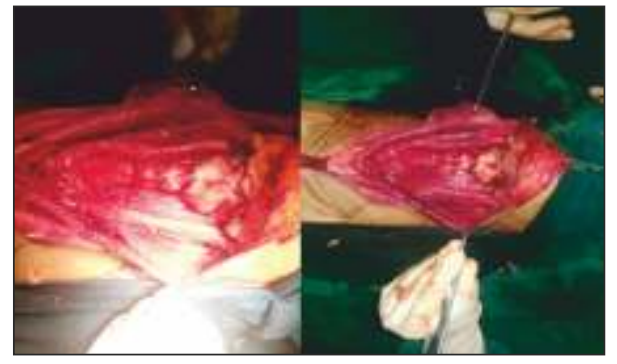

Intaoperative Picture

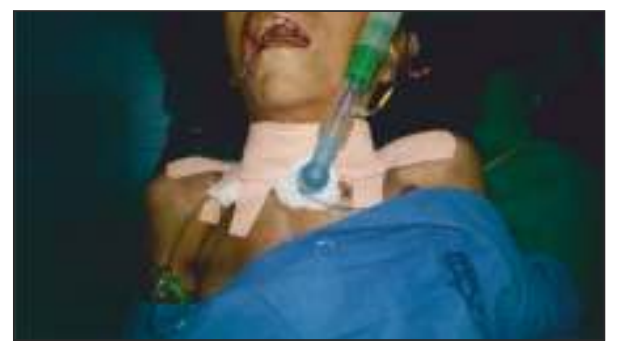

Postoperative Picture With Tracheostomy

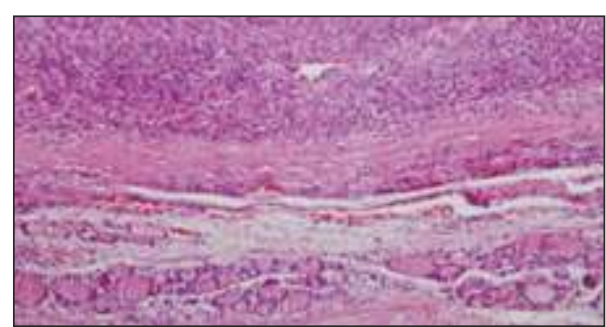

Histopathology Picture 


\section{Discussion}

Primary smooth muscle tumours of Thyroid gland (leiomyomas and leiomyosarcomas) are rarely seen (7).Of which primary thyroid leiomyosarcomais an extremely rare soft tissue cancer. Primary thyroid leiomyosarcoma represents just $0.014 \%$ of primary thyroid cancers (15) and is associated with an extremely aggressive clinical course, leading to an extremely poor 5-year survival rate. Primary Thyroid leiomyosarcoma is a mesenchymal malignant tumour with smooth muscle differentiation, arising from the smooth muscle cells of the vessels located in the thyroid capsule (2). Instead of metastasizing to regional lymph nodes $(7,9)$, leiomyosarcoma appears to invade adjacent tissue.Thus radical surgery is essential $(7,8,9,11,12,14)$. Previous studies have shown that, in patients with a large or locally aggressive tumour, the more aggressive the procedure, the more beneficial it is $(13,14)$. In hematoxylin-eosin staining, the tumour is composed of characteristic spindle shaped cells with hyperchromatic, blunt-ended nuclei and abundant eosinophilic cytoplasm which are in disordered fascicular pattern (5). The main differential diagnosis includes anaplastic thyroid

\section{References}

1. Tran LM, Mark R, M eier R, Calcaterra TC, Parker RG: Sarcomas of the head and neck: prognostic factors and treatment strategies. Cancer 1992, 70:169-177

2. Amal B, Fatemi HE, Souaf I, M oumna K, Affaf A. A rare primary tumor of the thyroid gland: report a new case of leiomyosarcoma and literature review. Diagnostic Pathology.2013, 8:36

3. Ozaki O, Sugino K, Mimura T, Ito K, Tamai S, Hosoda Y. Primary Leiomyosarcoma of the Thyroid Gland. Japan J Surg. 1997, 27:177-180

4. Mansouri H, Gaye M, Errihani H, Kettani F, Gueddari IE. Leiomyosarcoma of the thyroid gland.ActaOto-Laryngologica.2008, 128: 335-336

5. Ege B, LeventogluS.Primaryleiomyosarcoma of the thyroid. J Korean Surg Soc. 2013, 85:43-46

6. Nemenqani D, Yaqoob N, Khoja H. Leiomyosarcoma M etastatic to the Thyroid Diagnosed by Fine Needle Aspiration Cytology. J Pak Med Assoc. 2010, 60:307-309

7. Ozaki O, Sugino K, M imura T, Ito K, Tamai S and Hosoda Y: Primary leiomyosarcoma of the thyroid gland. Surg Today 27: 177-180, 1997

8. Takayama F, Takashima S, M atsuba H, Kobayashi S, Ito N and Sone S: MR imaging of primary leiomyosarcoma of the thyroid gland. Eur J Radiol 37: 36-41, 2001 carcinomas, solitary fibrous tumours, spindle cell tumours with thymus-like differentiation, medullary carcinoma and other sarcomas (2). Primary thyroid leiomyosarcoma usually displays smooth muscle cells with histological features characterized by atypical pleomorphism, prominent mitotic activity, necrosis, haemorrhage, and invasive and/or extra thyroidal growth (5).

\section{Conclusion}

A diagnosis of Leiomyosarcoma is dependent on the presence of smooth muscle actin, which may be identified by immunohistochemical staining. This rare malignant nonepithelial tumour of the thyroid gland occurred in older patients with a median age of 65 years old $(3,4)$. Immunohistochemical analysis is a gold standard for diagnosis. Although the primary treatment of primary thyroid leiomyosarcomais surgical excision (2),adjuvant radiotherapy and chemotherapy should be considered in high grade tumours. The treatment protocols for primary thyroid leiomyosarcomahave not been well established yet, because of their rareness and poor prognosis. We believe that aggressive therapeutic approach is necessary for aggressive cancers.

9. Day AS, Lou PJ, Lin WC and Chou CC: Over-expression of c-kit in a primary leiomyosarcoma of the thyroid gland. Eur Arch Otorhinolaryngol 264: 705-708, 2007.

10. Mansouri H, Gaye M, Errihani H, Kettani F and Gueddari BE: Leiomyosarcoma of the thyroid gland. ActaOtolaryngol 128: 335-336, 2008

11. Amal B, El Fatemi H, Souaf I, M oumna K and Affaf A: A rare primary tumor of the thyroid gland: Report a new case of leiomyosarcoma and literature review. DiagnPathol 8: 36, 2013.

12. Ege B and Leventoglu S: Primary leiomyosarcoma of the thyroid. J Korean SurgSoc 85: 43-46, 2013.

13. M ouaqit $\mathrm{O}$, Belkacem Z, Ifrine $\mathrm{L}, \mathrm{M}$ ohsine $\mathrm{R}$ and Belkouchi A: A rare tumor of the thyroid gland: Report on one case of leiomyosarcoma and review of literature. Updates Surg 66: 165-167, 2014

14. Tanboon J and Keskool P: Leiomyosarcoma: A rare tumor of the thyroid. EndocrPathol 24: 136-143, 2013

15. Thompson LD, Wenig BM, Adair CF, Shmookler BM and Heffess CS: Primary smooth muscle tumors of the thyroid gland. Cancer 79: 579-587, 1997. 